\title{
Reparative Bone Formation with the Replacement of Experimental Cavity Defect of the Bone with a Mesh of Titanium Nickelide Abstract
}

\author{
Irianov Yu $\mathbf{M}^{1}$ and Kiryanov $\mathrm{NA}^{2 *}$ \\ ${ }^{1}$ Russian Ilizarov Scientific Center for Restorative Traumatology and Orthopedic, \\ Russia \\ ${ }^{2}$ Izhevsk State Medical Academy, Russia
}

\section{Research Article \\ Volume 2 Issue 1}

Received Date: December 26, 2017

Published Date: January 31, 2018

*Corresponding author: Prof Nikolay Kiryanov, Izhevsk State Medical Academy, Kommunarov str., 281, Russia, Tel: +7 912 850-25-89; E-mail: kirnik@list.ru

\section{Abstract}

Purpose of Study: To study reparative osteogenesis and tissue integration characteristics for implanting threedimensional mesh structures of titanium nickelide into a bone cavitary defect.

Material and Methods: The authors modeled cavitary defects of femoral metaphysis experimentally in Wistar rats divided into an experimental group and control one. The study duration was 60 days in total. The methods of radiography, those of light and electron microscopy, X-ray electron probe microanalysis used. Results: Under implantation the defect was filled with cancellous bone the volumetric density of which more than 1,5-fold exceeded control values $(p<0.001)$. The implant had biocompatibility, osteoconductive and osteoinductive properties, it stopped inflammatory processes. The membrane protective barrier which prevented connective tissue sprouting was formed on the implant surface in the defect periosteal zone. The osteointegrative junction was formed being persisted up to the end of the experiment. Reparative osteogenesis was performed by direct intramembranous and apposition type.

Conclusion: The implant of three-dimensional mesh titaium-nickelide structures has marked osteoplastic properties, and it can be successfully used in orthopedic surgery.

Keywords: Reparative Osteogenesis; Bone defect; Implant; Titanium nickelide

\section{Introduction}

Connective tissue ingrowth from the periosteal surface is the main obstacle for restitution of large-volume bone defects that is caused by a higher rate of migrating fibroblasts comparing with osteogenic cells [1-3]. This can inhibit reparative osteogenesis process completely or partially, as well as be a cause of the defect filling with 
dense connective tissue of scarry type. In order to create optimal conditions for formation of organotypic regenerated bone the technique of guided reparative osteogenesis was developed using the membrane technology which prevented connective tissue sprouting $[4,5]$. The membranes of synthetic and natural materials are used for this purpose which, however, are not osteointegrated, they can cause an inflammatory reaction and tissue swelling, in case of their use a repeat surgical intervention is required [6,7]. New possibilities have arisen due to introducing medical technologies related to using the implants based on nickel and titanium which are approached to bone tissue by their mechanic characteristics and are biocompatible [8-11].

The purpose of this work is to study reparative osteogenesis morphological features when implanting mesh structures of titanium nickelide into a cavitary defect of femoral metaphysis.

\section{Materials and Methods}

Cavitary defects of femoral metaphysis modeled in adult male Wistar rats in the experimental group $(n=20)$ and control one $(n=20)$; the defect volume was $0.02 \mathrm{~cm}^{3}$ that amounted for about $40 \%$ of total metaphysis volume. The volume of the metaphysis was determined by the volume of water displaced by it. The defect was filled with water using a measuring syringe and its volume. All the manipulations were performed according to the Ministry of Health Order No 708 of 23.08.2010 "Approval of the rules of good laboratory practice". The implant was introduced into the defect in the animals of the experimental group, no additional manipulations performed in the control group. The implant was a mesh framework made of nickelide-titanium thread (TH-10 brand of $90 \mu \mathrm{m}$ caliber) formed by the type of knitting with cells - through open pores of 100-300 $\mu$ m diameter [12]. The thread was made of composite material comprising a core of nanostructured monolithic titanium nickelide and a titanium-oxide microporous surface layer of 5-7 $\mu \mathrm{m}$ (Certificate No POOCRU.AЯ79H18304). The animals were withdrawn from the experiment after 7,14 , 30 and 60 days (five animals were used for each time point). The bone meta-epiphyseal zone was sewn out, fixed in $2 \%$ paraformaldehyde and glutaraldehyde solution, embedded in araldite and paraffin (after decalcification). Histological sections were stained with hematoxylin, eosin and by Van Gieson. The araldite blocks studied using INCA-200 Energy X-ray electron probe micro-analyzer ("Oxford Instruments", England) and JSM840 scanning electron microscope ("Jeol", Japan). With the help of this device, images (maps) were obtained in the characteristic X-ray radiation of calcium atoms. The structures of bone tissue were visualized and the necessary measurements were made. The bulk density of bone tissue was determined (in\%). As the ratio of the area occupied by bone structures in the image to the total area of the map. The bone tissue compactness index (ratio of bone structure and non-mineralized components) and concentration of osteotropic macronutrients (calcium, phosphorus, magnesium, sodium and sulfur) in the bone regenerate were calculated $[10,11]$.

\section{Methods of a Statistical Analysis}

Made using "Microsoft Excel - 2010" Program. The data were presented as mean value $(M)$, error of representation $(m)$ and level of difference significance $(p)$. The significance of the intergroup differences of the compared parameters in view of normal distribution was calculated using Student t-test. The differences considered statistically significant at $\mathrm{p}<0.05$.

\section{Results}

The process of bone formation which occurred from periosteum, endosteum, bone marrow and the damaged bone structures of the defect edges was observed in the both groups of animals 7, 14 days after surgery. In the control group of animals, a non-matured regenerated bone (of connective-tissue type) was formed in the periosteal zone, its collagen fibers grew from periosteum into the defect central zone as strip-like bundles. The defect was filled with loose connective and granulation tissue where the foci of lymphocytic and plasmacytic infiltration and fibrin clots were located. The islets of reticulofibrous bone tissue represented as a fine-cellular network of interwoven bone-osteoid trabeculae were formed near the defect outer edges in the periosteum, as well as in the endosteum. Randomly arranged fragments of the damaged bone structures, as well as separate unrelated foci of newly formed bone tissue as short boneosteoid trabeculae lining the defect inner surface located near the defect inner edges. The bone tissue volumetric density in the defect and the compactness index of the regenerated bone amounted to about 30\% (p < 0.001$)$ of the intact metaphysis values 14 days after surgery in the control group of animals (Table 1).
Irianov Yu M and Kiryanov NA. Reparative Bone Formation with the Replacement of Experimental Cavity Defect of the Bone with a Mesh of Titanium Nickelide Abstract. J Human Anat 2018, 2(1): 000120.
Copyright $($ Irianov Yu M and Kiryanov NA. 


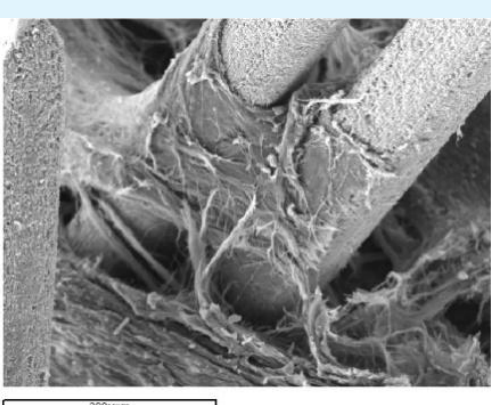

a

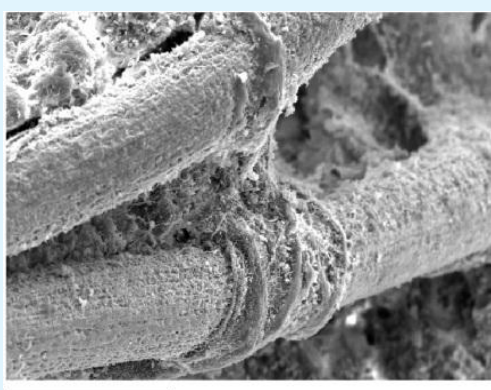

b

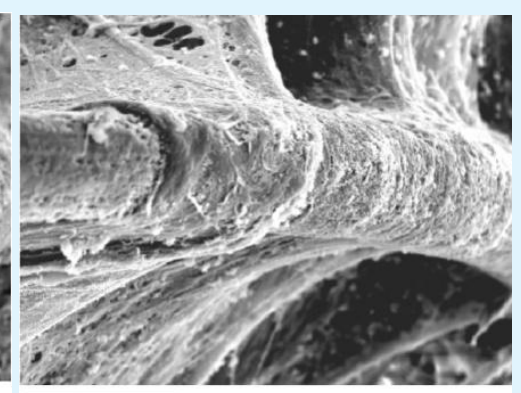

C

Figure 1: Connective-tissue cover on the implant surface in the defect periosteal zone 7 (A) and 14 (B, C) days after surgery. Scanning electron microscopy, magnification $\times 160$.

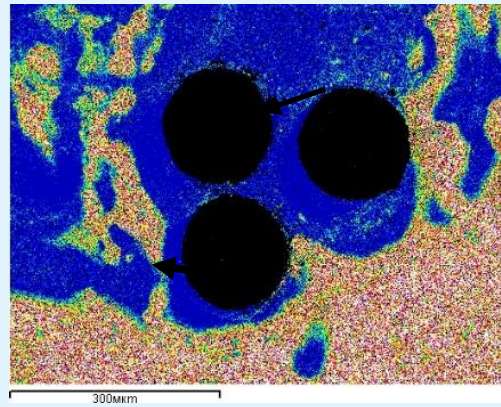

a

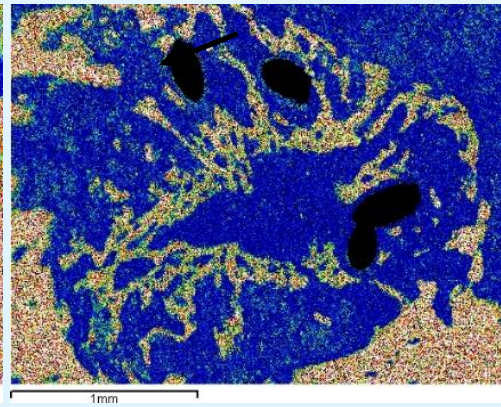

b

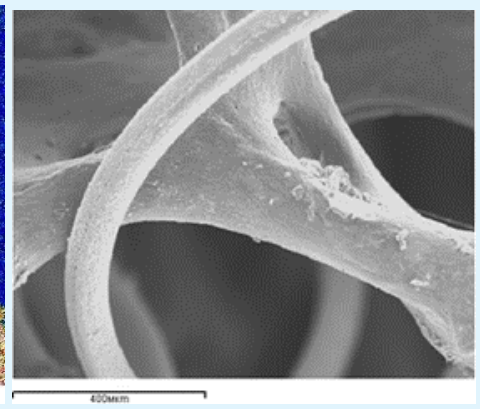

C

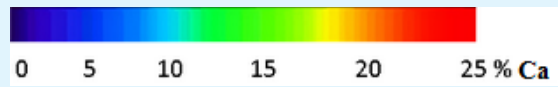

Figure 2: Reparative osteogenesis in the metaphyseal defect 7 (a) and 14 (b, c) days after surgery, arrows indicate the areas of osteointegration. $a, b$ - charts of x-ray electron probe microanalysis, images in calcium characteristic x-ray emission, $c$ - scanning electron microscopy (organic components removed with 6\% sodium hypochlorite solution), magnification: $\mathrm{a}-\times 100, \mathrm{~b}-\times 25, \mathrm{c}-\times 70$.

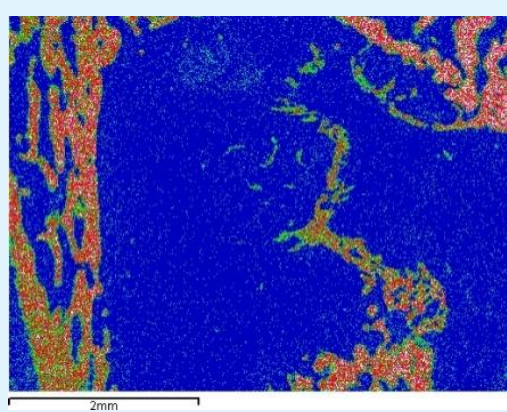

a

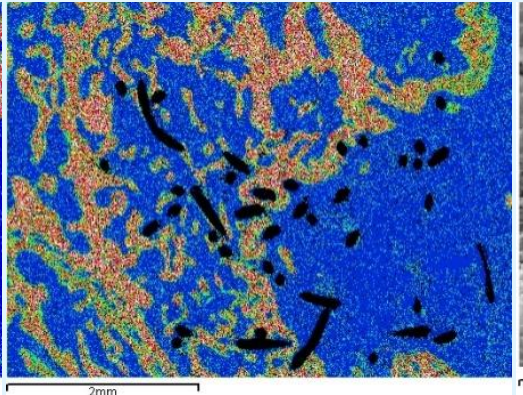

b

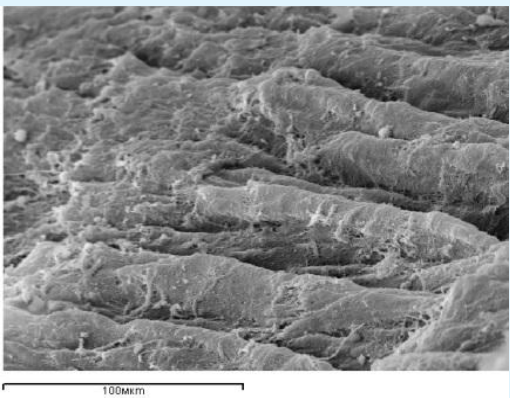

C

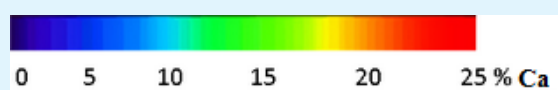

Figure 3: The metaphyseal defect filling 30 days after surgery in the control (a) and experimental (b, c) group of animals, $a, b$ - charts of x-ray electron probe microanalysis, images in calcium characteristic $\mathrm{x}$-ray emission, magnification $\times 20$; $c-$ scanning electron microscopy, magnification $\times 500$.

Irianov Yu M and Kiryanov NA. Reparative Bone Formation with the Replacement of Experimental Cavity Defect of the Bone with a Mesh of Titanium Nickelide Abstract. J Human Anat 2018, 2(1): 000120.
Copyright@ Irianov Yu M and Kiryanov NA. 
A thin membrane-like cover of connective tissue was formed round the implant threads and cells in the defect periosteal zone in the experimental group animals 7, 14 days after surgery (Figure 1a). The cover formation began on the surface of the thread in the places of its weavings and spread from the periphery of the cells to their center. The cover had a layered structure. The inner layer consisted of dense formalized connective tissue. Collagen fibers were collected into dense circularly oriented lacelike bundles, and they braided the implant threads in the form of a sleeve, they were firmly fixed to their microporous surface, grew into the gaps between them and provided the fixation of the implant threads both between each other and in the bone defect (Figure 1b). The outer layer of the cover was formed with dense nonformalized connective tissue the thin flat wavy twisted collagen-fiber bundles of which located as a fine-cellular network between the lace-like bundles of the inner layer framework and connected with surrounding bone and connective-tissue structures (Figure 1c). The areas of active appositional bone formation were observed below the connective-tissue cover in the endosteal and central zone of the defect and at its edges round the implant structures, as well as on their surface. A layer of reticulofibrous bone tissue of 300-400 $\mu \mathrm{m}$ thickness emerged on the implant thread surface directly forming osteointegration connection (Figure 2a). The implant threads in the osteointegration areas were coated with mineralizing bone matrix. Newly formed trabeculae grew into the implant fine-cellular structure (Figures $2 \mathrm{~b} \& 2 \mathrm{c}$ ). The results of quantitative studies (Table 1) evidenced of reparative osteogenesis significant activation, as well as of increasing the maturity degree of newly formed bone tissue in the regenerated bones of the animals of the experimental group comparing with those in the control group. Thus, bone tissue volumetric density in the defect was $144.19 \%$, index of compactness - $155.56 \%$ in comparison with the values in the animals of the control group $(\mathrm{p}<0.001)$.

The defect was filled with regenerated bone 30-60 days after surgery in the control group of animals, where littlemineralized dense non-formalized connective tissue growing into from the periosteal surface prevailed (Figure 3a). The initial stages revealed for periostealintermediary uniting and forming cortex resembling cancellous bone by structure. The operated metaphyseal zone acquired a marked conical shape. Little-calcified dense connective tissue prevailed in the periosteal zone of the regenerated bone. The fine-cellular bone structures of the periosteal regenerated bone fused with endosteal newly formed trabeculae, arcuately grew into the defect central zone and formed a thin crescent layer of newly formed cortical bone. Osteogenesis foci were observed in the defect central and marginal zones where osteoid areas revealed, as well as fragments of newly formed littlemineralized reticulofibrous bone trabeculae isolated from each other by wide interlayers of loose connective tissue with the cavities filled with lymphocytic and macrophage elements. Bone tissue volumetric density in the defect, index of compactness of the regenerated bone, the content of calcium and phosphorus amounted to $50-60 \%$ of the intact metaphysis values $(\mathrm{p}<0.001)$ (Tables $1 \& 2)$.

\begin{tabular}{|c|c|c|c|c|c|c|c|}
\hline \multirow[b]{2}{*}{ Parameters } & \multicolumn{6}{|c|}{ Period of the experiment, days } & \multirow{3}{*}{ Meta-physis } \\
\hline & \multicolumn{2}{|c|}{14} & \multicolumn{2}{|c|}{30} & \multicolumn{2}{|c|}{60} & \\
\hline \multirow{5}{*}{ Bone tissue, \% Index of compact-ness } & Cont & Exp & Cont & $\operatorname{Exp}$ & Cont & $\operatorname{Exp}$ & \\
\hline & $8.443 \pm$ & $12.174 \pm$ & $13.543 \pm$ & $19.764 \pm$ & $15.022 \pm$ & $22.984 \pm$ & $25.072 \pm$ \\
\hline & 0.381 & 0.6131 & 0.681 & $0.962^{1}$ & 0.744 & 1.0941 & 1.212 \\
\hline & $0.092 \pm$ & $0.143 \pm$ & $0.164 \pm$ & $0.252 \pm$ & $0.181 \pm$ & $0.310 \pm$ & $0.333 \pm$ \\
\hline & 0.005 & $0.012^{3}$ & 0.013 & $0.021^{2}$ & 0.013 & $0.021^{2}$ & 0.024 \\
\hline
\end{tabular}

Table 1: Bone tissue volumetric density in the metaphysis defect in the animals from the control group (Cont) and the experimental one (Exp) and in the intact metaphysis of the contralateral limb $(\mathrm{M} \pm \mathrm{m})$.

Note: ${ }^{1,2,3}$ intergroup differences $\left({ }^{1} \mathrm{p}<0.001,{ }^{2} \mathrm{p}<0.01,{ }^{3} \mathrm{p}<0.05\right)$.

\begin{tabular}{|c|c|c|c|}
\hline Elements & Control $(\mathbf{n}=\mathbf{5})$ & Experiment $(\mathbf{n}=\mathbf{5})$ & Metaphysis of contralateral limb (n=5) \\
\hline Sodium & $0.41 \pm 0.02$ & $0.44 \pm 0.02$ & $0.40 \pm 0.02$ \\
\hline Magnesium & $0.22 \pm 0.01$ & $0.25 \pm 0.01$ & $0.22 \pm 0.01$ \\
\hline Phosphorus & $1.93 \pm 0.04$ & $2.94 \pm 0.13^{1}$ & $3.22 \pm 0.15$ \\
\hline Sulfur & $0.23 \pm 0.01$ & $0.30 \pm 0.02^{2}$ & $0.22 \pm 0.01$ \\
\hline Calcium & $3.85 \pm 0.16$ & $5.89 \pm 0.26^{1}$ & $6.44 \pm 0.31$ \\
\hline
\end{tabular}

Table 2: Content of osteotropic chemical elements in the regenerated bone of the control and experimental groups of animals 60 days after surgery and in the intact metaphysis of the contralateral limb $(M \pm m, \%)$.

Note: ${ }^{1,2}$ intergroup differences $\left({ }^{1} \mathrm{p}<0.001,{ }^{2} \mathrm{p}<0.05\right)$.

Irianov Yu M and Kiryanov NA. Reparative Bone Formation with the Replacement of Experimental Cavity Defect of the Bone with a Mesh of Titanium Nickelide Abstract. J Human Anat 2018, 2(1): 000120.
Copyright $($ Irianov Yu M and Kiryanov NA. 
In the experimental group of animals, the area of bone defect was filled with regenerated bone where cancellous bone prevailed 30-60 days after surgery (Figure 3b). A new area of cortical layer was formed represented as compact bone of lamellar structure. The bundles of collagen fibers of the protective cover dense connective tissue located on the defect periosteal surface round the implant which formed the interweaving of village fence type (Figure 3c). The implant threads were surrounded by osteoid or they were completely overgrown by newly formed bone tissue thereby forming a composite compact bone reinforced by titanium nickelide. Bone tissue volumetric density, index of compactness and mineralization degree of the regenerated bone were somewhat less comparing with the intact metaphysis values, but these differences were not statistically significant by the end of the experiment, and, at the same time, they more than 1.5 -fold exceeded $(p<0.001)$ the values in the control group of animals (Tables 1 and 2).

\section{Discussion}

Substitution or reconstruction of extensive bone cavity defects caused by congenital or acquired pathology is an actual medical and social problem The main methods of surgical treatment are variants of bone plastic with the use of various synthetic, biological and composite materials. However, when studying the long-term results, it was found that most of these materials are not osseointegrated, but surrounded by a fibrous capsule. The use of own bone (autotransplantation) is associated with an additional traumatic effect and is limited by the inability to take the necessary amount of autologous bone material, especially in children. In this case, there is a risk of transmission of various diseases and the development of a number of serious complications of the immune nature, which is often accompanied by graft rejection and suppuration in the postoperative period [13]. The results of this study showed that the implant studied well performed the defect form, had good biocompatibility, expressed osteoconductive properties. The microporous structure of the surface layer of the implant filaments ensured the adhesion of the cells of the regenerate and the formation of the osseointegrative compound, which was maintained until the end of the experiment. The development of osteogenic differentiation of cells on the surface of the implant was proved by the development of a specific calcified matrix. Regenerate tissues and blood vessels easily germinated into the implant without disrupting the integrity of the implant. In the periosteal region of the defect, a layer of dense connective tissue was formed on the surface of the implant, which served as a biological protective barrier preventing the germination of paraosal connective tissue. The defect was compensated by a spongy bone, the bulk density of which at all stages of the experiment was more than one and a half times higher than the control indices, and its mineral composition approximated the parameters of the spongy bone of the intact metaphysis. Reparative bone formation was carried out by the type of direct intramembranous osteogenesis. In none of the cases there were signs of an inflammatory process, which confirmed the data we received earlier [10]. For the first time during the implantation, an artificial composite biological tissue reinforced with titanium nickelide filaments was obtained: dense fibrous connective tissue, spongy and compact bone. The fine-celled structure and microporosity of the surface of the implanted structures created capillary properties, due to which adsorption (mechanical impregnation) of endogenous bone morphogenetic proteins (BMP) and growth factors (cytokines) occurred. The functional activity of the latter, providing proliferation and accelerated differentiation of osteogenic cells, stimulating the synthesis of collagen, osteocalcin, alkaline phosphatase, activating the mineralization of the organic matrix of bone $[13,14]$, ensured the osteoinductiveness of the implant.

\section{Conclusion}

The implant made of mesh structures of titanium nickelide is an effective osteoconductor and osteoinductor, provides prolonged activation of reparative bone formation and spatial development of bone tissue in the defect. Atraumatism of surgical intervention, absence of biological rejection reaction place the implant in the range of the most optimal osteoplastic materials, and its application seems theoretically grounded and promising, especially in patients with reduced osteogenetic and reparative potential, including in mature and elderly patients, as well as in children.

\section{References}

1. Jie L, Kerns DG (2014) Mechanisms of Guided Bone Regeneration: A Review. Open Dent J 8: 56-65.

2. Kim JY, Yang BE, Ahn JH, Park SO, Shim HW (2014) Comparable efficacy of silk fibroin with the collagen membranes for guided bone regeneration in rat calvarial defects. J Adv Prosthodont 6(6): 539-546.

3. Jung RE, Fenner $\mathrm{N}$, Hämmerle $\mathrm{CH}$, Zitzmann $\mathrm{NU}$ (2013) Longterm outcome of implants placed with guided bone regeneration (GBR) using resorbable and non-resorbable membranes after 12-14 years. Clin Oral Implants Res 24(10): 1065-1073. 
4. Hämmerle $\mathrm{CH}$, Jung RE (2003) Bone augmentation by means of barrier membranes. Periodontol 33: 36-53.

5. Karring T, Nyman S, Gottlow J, Laurell L (2000) Development of the biological concept of guided tissue regeneration-animal and human studies. Periodontol 1: 26-35.

6. Schmidmaier G, Baehr K, Mohr S, Kretschmar M, Beck $S$, et al. (2006) Biodegradable polylactide membranes for bone defect coverage: biocompatibility testing, radiological and histological evaluation in a sheep model. Clin Oral Implants Res 17(4): 439-444.

7. Leeuwen AC, Huddleston Slater JJ, Gielkens PF, Jong JR, Grijpma DW, et al. (2012) Bos RR Guided bone regeneration in rat mandibular defects using resorbable poly (trimethylene carbonate) barrier membranes. Acta Biomater 8(4): 1422-1429.

8. Chernov VF, Bevzyuk AN, Chernov AV, Iryanov YM (2008) The study of the implantation of mesh structures of NiTi. Med Sci Edu Urals 2: 98.

9. Iryanov $\mathrm{Yu} \mathrm{M}$, Iryanova TYu (2012) Bone defect replacement under condition of transosseous osteosynthesis and titanium nicelide implant application. Morphology 4: 83.

10. Iriyanov $\mathrm{Yu} \mathrm{M}$, Chernov VF, Radchenko SA, Chernov AV (2013) Plastic efficiency of different implants used for repair of soft and bone tissue defects. Bull Exp Biol Med 155(4): 518-521.

11. Irianov YM, Diuriagina OV, Karaseva TY, Karasev EA (2014) The osteoplastic effectiveness of the implants made of mesh titanium nickelide constructs. Bosn J Basic Med Sci 14(1): 4-7.

12. The implant to replace bone defect RF Pat 111759 (Russian Ilizarov Scientific Center «Restorative Traumatology and Orthopedics») Bul 36(2011): 1.

13. Uebersax L, Hagenmüller $H$, Hofmann $S$, Gruenblatt $E$, Müller R, et al. (2006) Effect of scaffold design on bone morphology in vitro. Tissue Eng 12(12): 34173429.

14. Osyczka AM, Diefenderfer DL, Bhargave G, Leboy PS (2004) Different effects of BMP-2 on marrow stromal cells from human and rat bone. Cells Tissues Organs 176(1-3): 109-119. 